\title{
Editorial: Dementia, Frailty and Aging
}

\author{
Wee-Shiong Lim ${ }^{1 *}$, Marco Canevelli ${ }^{2}$ and Matteo Cesari ${ }^{3,4}$ \\ ${ }^{1}$ Department of Geriatric Medicine, Institute of Geriatrics and Active Ageing, Tan Tock Seng Hospital, Singapore, Singapore, \\ ${ }^{2}$ Department of Human Neuroscience, Sapienza University of Rome, Rome, Italy, ${ }^{3}$ UOSD Geriatria, Fondazione IRCCS Ca' \\ Granda - Ospedale Maggiore Policlinico, Milan, Italy, ${ }^{4}$ Dipartimento di Scienze Cliniche e di Comunità, Università di Milano, \\ Milan, Italy
}

Keywords: dementia, frailty, aging, population aging, big data

\section{Editorial on the Research Topic}

\section{Dementia, Frailty and Aging}

Population aging is both a worldwide success story and a worldwide health conundrum, with the increasing age of populations around the world leading to unprecedented challenges (1). According to the United Nations report on World Population Prospects (2017), there is an estimated 962 million people aged 60 years and above who comprise $13 \%$ of the global population (2). The beginning of the twenty-first century has seen health systems worldwide struggling to deliver quality healthcare amidst challenges posed by aging populations (3). Traditional medicine and models of care have been premised on the evaluation and treatment of standalone and usually acute diseases occurring in relatively younger individuals. This contrasts with the current reality of multiple, interacting, and often chronic conditions affecting older persons. It is thus necessary to disentangle the pathophysiological mechanisms, clinical manifestations, and inter-relationships of age-related conditions in order to personalize clinical interventions and realign health systems to better address the unmet needs of frail older persons $(4,5)$.

Against this backdrop, frailty and dementia have emerged as priority areas in both research and clinical settings due to their high prevalence, impact on the individual's quality of life, and public health impact (6-8). These conditions aptly reflect the complexity of age-related pathological conditions, causally underpinned by a myriad of heterogeneous, interacting, and often unclear pathophysiological processes. Indeed, a hallmark of both conditions is the inherent difficulty in differentiating the effects of the normal aging process from the eventual pathophysiological deviations of the underlying disease $(9,10)$. Their occurrence and trajectories over time are strongly affected by a wide array of factors and determinants that are not confined to single biological systems and/or health domains (10). Moreover, environment and social factors also substantially influence the definition of different phenotypes. This raises the clarion call for a broader, integrated, and holistic approach that is able to more adequately capture the biological, clinical, and psychosocial complexities of frailty and dementia, thus paving the way for improvement in the consequent outcomes (11-13).

The present Research Topic represents a timely addition to the burgeoning body of evidence which aims to provide fresh perspectives in our understanding of the frailty and dementia phenomena occurring with aging. An area of particular interest is the emerging construct of cognitive frailty (CF), which is designed to operatively capture the co-existence of frailty and cognitive impairment in the absence of dementia (14). Using a modified version of the IANA/IAGG criteria (15), Ma et al. reported a $2.7 \%$ prevalence of CF in a Chinese older population. Older persons, women, and people living in rural areas were found to be at higher risk of CF. Corroborating the recommendations of the Lancet Commission report (7), depression and hearing impairment were independently associated with CF in elderly individuals with physical frailty. The study by Nyunt et al. explored the physical frailty phenotype in mild cognitive impairment (MCI). When compared with participants with "normal high cognition," there was a higher prevalence of frailty and pre-frailty attributable to low lean mass, slow gait speed, or balance and gait impairment. In their 5-year observational study of 91 subjects with amnestic MCI, 
Trebbastoni et al. also noted that frailty was associated with increased risk of conversion to Alzheimer's dementia, even in those with high baseline level of cognitive performance.

Four papers in this Research Topic shed further insights to illuminate the knowledge gap in our understanding of the interface between cognitive and physical domains. In their study of 269 elderly individuals with subjective memory complaints, Hooper et al. did not find any significant cross-sectional associations between fatigue and $\mathrm{A} \beta$ load. However, sensitivity analysis revealed a weak association with increased $A \beta$ in the hippocampus in subjects with $\mathrm{MCI}$, thereby providing indirect support for the construct of CF at the early stages of Alzheimer's disease. Chhetri et al. proposed the motoric cognitive risk (MCR) syndrome, characterized by the simultaneous presence of gait disturbances and memory complaints in older adults, as a means to examine the close interactions between cognitive and physical domains and identify individuals at risk of dementia and other age-related adverse outcomes. By summarizing the existing evidence from both human and animal models, Bellelli et al. highlighted the multiple common pathophysiologic mechanisms and pathways of delirium and frailty, to lay out the case for delirium as the cognitive harbinger of a state of frailty in the context of an acute clinical event. This opens the door for further studies to examine the contribution of physical frailty to adverse outcomes in delirium, and conversely, the deleterious impact of delirium on physical frailty (16). Using the examples of frailty and MCI, the review by Canevelli et al. challenged the widelyheld assumptions of these entities as unequivocally prodromal stages of a future disease state by providing a timely reminder of our incomplete understanding of the transitions of clinical atrisk conditions and their potential for clinical improvement and spontaneous reversion.

Dementia is a devastating and debilitating illness that has far-reaching public health, social and economic ramifications. Therefore, the Research Topic submissions also covered pertinent areas in dementia such as caregiving and diagnosis. To keep pace with the projected exponential rise in dementia, it is imperative that we tap upon the "invisible workforce" of family caregivers and understand the factors that predispose to caregiver burden (17). Li et al. confirmed the existence of the unique "worry about performance" (WaP) burden in the multidimensionality of caregiver burden beyond role and personal strain. Unlike other factors, WaP was significantly reported even in early cognitive impairment, suggesting its potential as a possible target for interventions aimed at improving self-efficacy among caregivers in the milder stages of burden (18). In their study examining the rapidly expanding group of caregivers of dementia in oldestold (CDOO), Win et al. reported these were mainly older adult children who experienced significant role and personal strain

\section{REFERENCES}

1. Callahan CM, Foroud T, Saykin AJ, Shekhar A, Hendrie HC. Translational research on aging: clinical epidemiology as a bridge between the sciences. Transl Res. (2014) 163:439-45. doi: 10.1016/j.trsl.2013.09.002 rather than $\mathrm{WaP}$ while caring for their oldest-old family members with more impaired cognitive and physical function. To address the challenges of under-detection of dementia in the primary care setting, Teixeira et al. described a potentially scalable multi-stage strategy for community detection that involved initial screening by health professionals to identify at-risk individuals for more comprehensive evaluation. With the recent release of the NIAAA Research Framework directed toward a biological definition of Alzheimer's disease (19), the real-world study of geriatrics outpatients by Dolci et al. highlighted the discrepancy between clinical diagnosis of Alzheimer's disease with cerebrospinal fluid and neuroimaging biomarkers, thereby reiterating the caution against premature and inappropriate usage of biomarker-based research frameworks in general medical practice.

Novel approaches are also suggested in this Research Topic. Reviving a 100 -year old idea about a possible role played by gut microbiota in modulating brain morphology and function across the life-course (20), Calvani et al. proposed the fascinating concept of the "second brain aging" which links age-related changes in the gut mircrobiota to neurodegeneration and related conditions (including depression, Alzheimer's disease, and Parkinson's disease). This raises the tantalizing prospect of developing interventions that target the gut microbiota as part of a comprehensive strategy in dementia prevention and treatment. Yatawara et al. explicated the cognitive-anatomical basis of getting lost behavior in patients with mild Alzheimer's disease. They reported that the top-down modulation deficit is localized to the medial temporal lobe and did not follow the typical mechanism in healthy aging, highlighting the need to target both working memory and visuospatial deficits simultaneously. Lastly, the thoughtful review by Lenca et al. explored the potential of harnessing big data approaches to improve current preventive and predictive models in dementia care and research (e.g., enabling earlier diagnosis, optimizing resource allocation, and delivering individualized treatments tailored to patients). The authors highlighted technical, scientific, ethical, and regulatory challenges and proposed the need for multi-level integrative approaches to chart the route ahead for research, ethics, and policy.

As guest editors for this research topic on frailty, dementia, and aging, we are delighted to commend to you the collection of 14 articles as an important contribution to "evidence-balanced medicine" in the real world of frail older persons (21).

\section{AUTHOR CONTRIBUTIONS}

W-SL, MarC, and MatC conceived the manuscript. W-SL drafted the paper. MatC critically appraised and edited the manuscript. All authors read and approved the final version of the paper.

2. United Nations report on World Population Prospects. World Population Prospects: The 2017 Revision, Key Findings and Advance Tables. United Nations DoEaSA, Population Division (2017). Available online at: https://esa. un.org/unpd/wpp/Publications/Files/WPP2017_KeyFindings.pdf (accessed September 25, 2017). 
3. Lim WS, Wong SF, Leong I, Choo P, Pang WS. Forging a frailty-ready healthcare system to meet population ageing. Int J Environ Res Public Health (2017) 14:1448. doi: 10.3390/ijerph14121448

4. Woo J. Designing fit for purpose health and social services for ageing populations. Int J Environ Res Public Health (2017) 14:457. doi: 10.3390/ijerph14050457

5. Cesari M, Prince M, Thiyagarajan JA, De Carvalho IA, Bernabei R, Chan P, et al. Frailty: an emerging public health priority. J Am Med Dir Assoc. (2016) 17:188-92. doi: 10.1016/j.jamda.2015.12.016

6. Beard JR, Officer A, de Carvalho IA, Sadana R, Pot AM, Michel J-P, et al. The World report on ageing and health: a policy framework for healthy ageing. Lancet (2016) 387:2145-54. doi: 10.1016/S0140-6736(15)00516-4

7. Livingston G, Sommerlad A, Orgeta V, Costafreda SG, Huntley J, Ames D, et al. Dementia prevention, intervention, and care. Lancet (2017) 390:2673734. doi: 10.1016/S0140-6736(17)31363-6

8. Dent E, Lien C, Lim WS, Wong WC, Wong CH, Ng TP, et al. The Asia-Pacific clinical practice guidelines for the management of frailty. J Am Med Dir Assoc. (2017) 18:564-75. doi: 10.1016/j.jamda.2017.04.018

9. Morley JE, Vellas B, Van Kan GA, Anker SD, Bauer JM, Bernabei R, et al. Frailty consensus: a call to action. J Am Med Dir Assoc. (2013) 14:392-7. doi: 10.1016/j.jamda.2013.03.022

10. Clegg A, Young J, Iliffe S, Rikkert MO, Rockwood K. Frailty in elderly people. Lancet (2013) 381:752-62. doi: 10.1016/S0140-6736(12)62167-9

11. Galvin JE. Prevention of Alzheimer's disease: lessons learned and applied. J. Am. Geriatr. Soc. (2017) 65:2128-33. doi: 10.1111/jgs.14997

12. Yaffe K. Modifiable risk factors and prevention of dementia: what is the latest evidence? JAMA Intern Med. (2018) 178:281-2. doi: 10.1001/jamainternmed.2017.7299

13. Walston J, Buta B, Xue Q-L. Frailty screening and interventions: considerations for clinical practice. Clin Geriatr Med. (2018) 34:25-38. doi: 10.1016/j.cger.2017.09.004

14. Canevelli M, Cesari M. Cognitive frailty: far from clinical and research adoption J Am Med Dir Assoc. (2017) 18:816-8. doi: 10.1016/j.jamda.2017.07.004
15. Kelaiditi E, Cesari M, Canevelli M, Van Kan GA, Ousset P-J, GilletteGuyonnet S, et al. Cognitive frailty: rational and definition from an (IANA/IAGG) international consensus group. J Nutr Health Aging (2013) 17:726-34. doi: 10.1007/s12603-013-0367-2

16. Chew J, Lim WS, Chong MS, Ding YY, Tay L. Impact of frailty and residual subsyndromal delirium on 1-year functional recovery: a prospective cohort study. Geriatr Gerontol Int. (2017) 17:2472-8. doi: 10.1111/ggi.13108

17. Gitlin LN, Marx K, Stanley IH, Hodgson N. Translating evidence-based dementia caregiving interventions into practice: state-of-the-science and next steps. Gerontologist (2015) 55:210-26. doi: 10.1093/geront/gnu123

18. Lim WS, Cheah WK, Ali N, Han HC, Anthony PV, Chan M, et al. Worry about performance: a unique dimension of caregiver burden. Int Psychogeriatr. (2014) 26:677-86. doi: 10.1017/S1041610213002445

19. Jack CR, Bennett DA, Blennow K, Carrillo MC, Dunn B, Haeberlein SB, et al. NIA-AA Research Framework: toward a biological definition of Alzheimer's disease. Alzheimers Dement. (2018) 14:535-62. doi: 10.1016/j.jalz.2018. 02.018 .

20. Mackowiak PA. Recycling Metchnikoff: probiotics, the intestinal microbiome and the quest for long life. Front Public Health. (2013) 1:52. doi: 10.3389/fpubh.2013.00052

21. Lim WS, Ding Y. Evidence-balanced medicine:"Real" evidence-based medicine in the elderly. Ann Acad Med Singapore (2015) 44:1-5.

Conflict of Interest Statement: The authors declare that the research was conducted in the absence of any commercial or financial relationships that could be construed as a potential conflict of interest.

Copyright (c) 2018 Lim, Canevelli and Cesari. This is an open-access article distributed under the terms of the Creative Commons Attribution License (CC $B Y)$. The use, distribution or reproduction in other forums is permitted, provided the original author(s) and the copyright owner are credited and that the original publication in this journal is cited, in accordance with accepted academic practice. No use, distribution or reproduction is permitted which does not comply with these terms. 